\title{
Superimposed Nano-scale Diffraction Gratings as High Capacity Barcodes for Biological and Chemical Applications
}

\author{
S. W. Birtwell ${ }^{a, *}$, G. S. Galitonov ${ }^{a}$, H. Morgan ${ }^{\mathrm{b}}$, \\ N. I. Zheludev ${ }^{\text {a }}$ \\ ${ }^{a}$ Optoelectronics Research Centre and \\ ${ }^{\mathrm{b}}$ School of Electronics and Computer Science, University of Southampton, \\ Southampton, SO17 1BJ, UK
}

\begin{abstract}
We describe a new non-contact high capacity optical tagging technique based on the use of nanostructured barcodes. The tags are generated from a number of superimposed diffraction gratings. With one-dimensional diffraction, capacity for up to 68,000 distinguishable tags has been demonstrated, with a theoretical capacity of up to $10^{9}$ tags. Extension into two dimensions increases this theoretical limit to $10^{21}$ tags.
\end{abstract}

Key words: Diffraction gratings, optical barcodes, biological applications, combinatorial chemistry

PACS: 42.79.Dj, 42.62.Be, 87.80.-y

The rapid advances in high throughput screening, combinatorial chemistry, genomic and proteomic sciences have stimulated dramatic development of new encoding strategies for bead-based assays. Several optical encoding methods are currently used in these applications (1), including fluorescence, IR (2), Raman $(3)$ and optical $(4 ; 5)$ tagging on microbeads. Methods using magnetic tagging are also being investigated (6). In particular, multicolor optical coding has been achieved by embedding quantum dots of zinc sulfide-capped cadmium selenide nanocrystals into beads $(7 ; 8 ; 9 ; 10)$ while patterns can also be written in fluorescently dyed beads by spatially selective photo-bleaching to create spatially selective fluorescent tags (11). Here we investigate a new method for encoding small beads, which allows for non-contact reading. The tagging * Corresponding author. Email: swb@phys.soton.ac.uk, Web: www.nanophotonics.org.uk 
technique is based on fabricating a nano-structured pattern on the surface of the particle, which is only a few microns in size. The pattern is read by detecting the spatial distribution of laser light diffracted by the tag. Encoding information on the tag therefore requires creating many different patterns which can produce large numbers of unique distributions of diffracted light. In the simplest implementation, the pattern is a miniature diffraction grating, where information is coded in the pitch or spatial dimensions of the grating. However, the encoding capacity of such a tag can be greatly increased by fabricating tags with several overlapping nano-scale gratings.
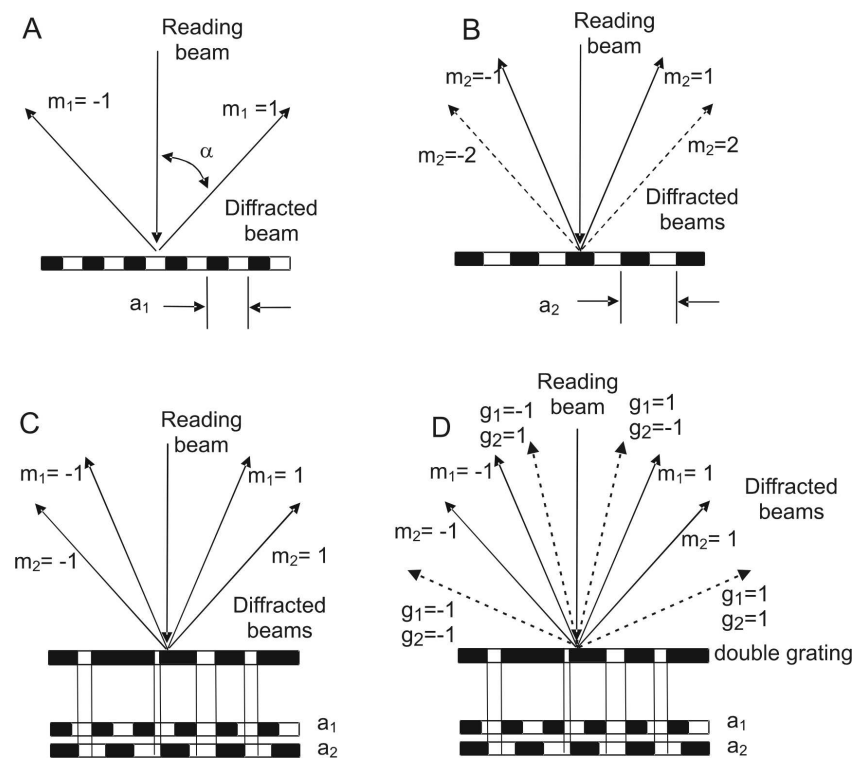

Fig. 1. A) first order diffraction from grating with pitch $a_{1}$; B) first and second order diffraction from grating with pitch $a_{2}$; C) first order diffraction from combinatorial grating made by superposition of gratings with pitches $a_{1}$ and $a_{2}$; D) first and ghost order diffraction from the combinatorial grating.

In a classical single diffraction grating, information may be encoded in the pitch or spacing of the grating, $a$. When such a grating is illuminated with light at wavelength $\lambda$, for example at normal incidence, a series of diffracted beams of different order $m$ is created, according to the equation $a \sin \alpha=m \lambda$. Here $\alpha$ is the angle of diffraction and $m$ is the diffraction order. Therefore, a measurement of the first order diffraction angle, at $m=1$, gives direct information about the pitch of the grating $a$. This principle is shown in figure 1. The number of distinguishable tags that can be manufactured depends on the ability to resolve a difference between two diffraction patterns. Two diffracted beams at diffraction angles $\alpha_{1}$ and $\alpha_{2}$ can be resolved if $\left|\alpha_{1}-\alpha_{2}\right| \geq \Delta \alpha_{1} / 2+\Delta \alpha_{2} / 2$, where $\Delta \alpha$ is angular width of the beam. Here $\Delta \alpha=\lambda(N a \cos \alpha)^{-1}$, where $N$ is the number of periods in the grating of length $L(N \approx L / a)$. Using this criterion it is possible to calculate the encoding capacity of diffractive barcodes with a single grating as a function of the grating length, by calculating the possible positions of the first order diffracted beam (neglecting all diffraction 
orders above $m=1$ ). The results for this are presented in fig. 2, curve (1).

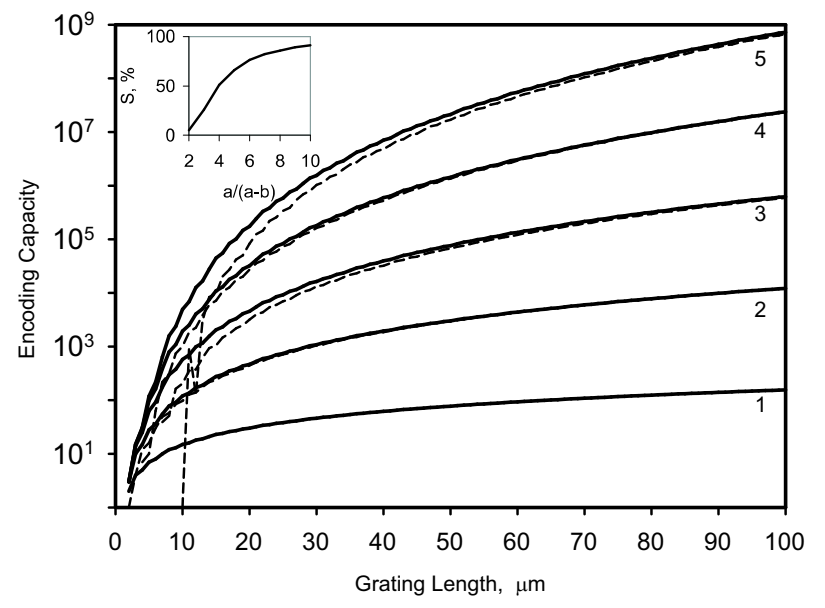

Fig. 2. Encoding capacity of a diffractive bar-code tag as a function of the length of the tag for different numbers of superimposed gratings. The number on each curve corresponds to the number of superimposed gratings. The inset show the level of intensity discrimination $S$ necessary to ensure higher order elimination, for different grating aspect ratios. Dashed lines show capacity after the elimination of tags with high intensity ghost beams.

A much larger capacity of tag can be obtained if several gratings are superimposed on the bead, as shown in fig. 1(C). Each of the superimposed gratings can be considered to produce its own set of diffracted beams. If the tag has $k$ superimposed gratings, the number of possible distinguishable codes $c$ is then given by the number of possible combinations of $k$ beams in the $n$ possible positions, using the combinatorial formula $c=\frac{n !}{(n-k) ! k !}$. The results of calculations of the tag capacity for several superimposed gratings using this resolution criteria is presented by the solid curves (2)-(5), fig.2. In practice the maximum number $k_{\max }$ of superimposed gratings in the tag which can be distinguished by diffraction is limited by the resolution $\delta$ of the tag's fabrication process and may be estimated as $k_{\max } \simeq \lambda /(2 \delta)$.

To further increase capacity, we can introduce '2-dimensional' tagging, using diffractive elements created from two perpendicular gratings. An example of such a tag is shown in the inset to fig. 3. The tags are read by the same method as the 1-dimensional tags, but now measuring the first order positions in the two perpendicular directions. The pitches of the two perpendicular gratings can be varied separately, and it is possible to have different numbers of superimposed gratings, $k_{x}$ and $k_{y}$, in each direction. The number of codes available for all possible combinations of pitches, with $k_{x}$ and $k_{y}$ values ranging from 1 up to a maximum $k$ is then

$$
c=\sum_{k_{x}=1}^{k} \sum_{k_{y}=1}^{k} \frac{n !}{\left(n-k_{x}\right) ! k_{x} !} \frac{n !}{\left(n-k_{y}\right) ! k_{y} !} .
$$


The results for the number of codes as a function of tag side length for a square two dimensional tag with different values of $k$, are shown in fig. 3. We can see from this that the maximum encoding capacity of tags $100 \mu \mathrm{m}$ in length is of the order of $10^{21}$ codes.

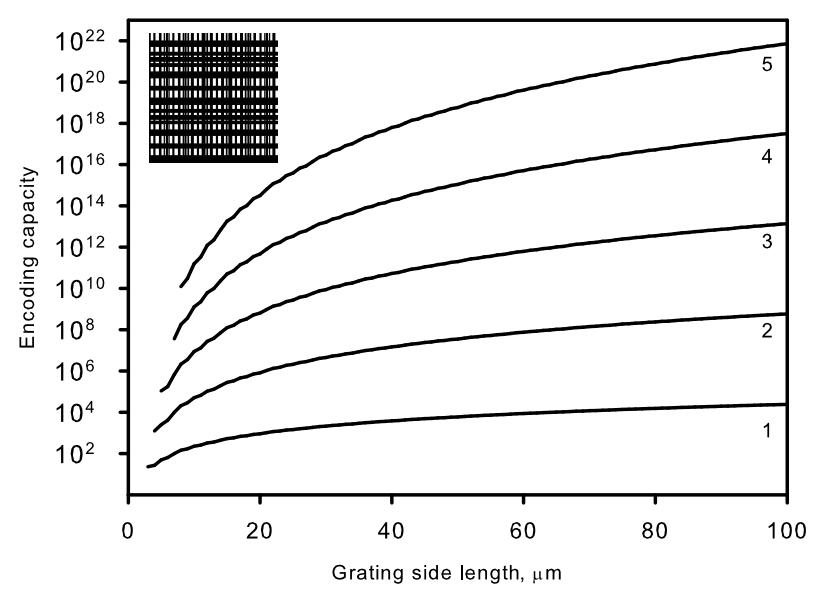

Fig. 3. Encoding capacity of a square 2-dimensional diffractive barcode tag as a function of the side length of the tag, for different values of $k$. The value of $k$ is denoted by the number on each curve. Inset shows an example 2-dimensional grating surface.

In order to demonstrate the 1-dimensional tagging concept, a chip library of chromium gratings was manufactured on a glass substrate using direct write electron beam lithography. The library of gratings contains almost 7,400 unique barcode tags, 50x50 $\mu \mathrm{m}$ separated by gaps of $200 \mu \mathrm{m}$. SEM images of these tags showing the range of different superimposed gratings are presented in fig. 6. With available nanofabrication minimum feature size $\delta$ of about $100 \mathrm{~nm}$ we have been able to demonstrate tags up to order three (containing three superimposed gratings) that are fully distinguishable by diffraction. This provides capacity for about 68,000 distinguishable tags. Higher order tags have also been fabricated, but they sometimes show failures in pattern reproduction which spoils the quality of diffraction (fig. 4 (iv)).

An example of the diffraction patterns created by these tags is presented in fig. 5. The gratings were 'read' using light from a HeNe laser (633nm) incident at normal angle to the sample. The diffraction pattern was observed on a screen parallel to the grating and captured using a CCD array detector. fig. 5(a) shows how the diffraction pattern changes with grating pitch; increasing in diffraction angle as the pitch decreases. The series of diffraction patterns (labeled A to $\mathrm{J}$ ) demonstrates how it is possible to uniquely distinguish between ten different tags containing only a single grating. In the photographs, the first order diffraction spots (marked $m_{1}=1$ as in fig. $1(\mathrm{~A})$ ) are highlighted by the solid circles, while the positions of the much weaker second order diffraction spots $\left(m_{1}=2\right)$ are indicated by dashed circles. 

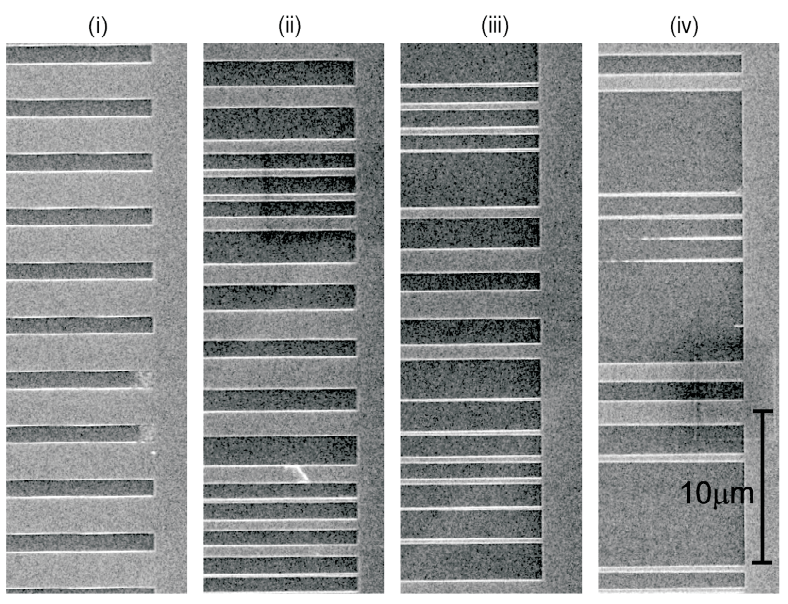

Fig. 4. SEM images of barcode tags of different order: (i) single grating tag; (ii) two superimposed gratings; (iii) three superimposed gratings; (iv) four superimposed gratings (note nano-lithography resolution limiting quality of this grating).

fig. 5(b) shows diffraction patterns from different tags containing three superimposed gratings (patterns HGF to QGF). Here, the first grating diffracts exactly as grating $\mathrm{G}$ in fig. 5(a), while the second grating diffracts as grating $\mathrm{F}$. The third grating differs from pattern to pattern. The first diffraction pattern is from a grating which diffracts as grating $\mathrm{H}$ in fig. 5(a), but then changes step by step to give a higher and higher diffraction angle. The tag code is formed from the letter labels of the individual grating on the tag; for example tag HGF in fig. 5(b) is formed from the gratings labelled $\mathrm{H}, \mathrm{G}$ and $\mathrm{F}$ seen in fig 5(a). In this way we have resolved diffraction patterns with up to three superimposed gratings. Further increase in the number of superimposed gratings on the tags led to increasing read errors due to the limited minimum feature size available in the grating fabrication process.

The analysis given so far assumes normal incidence of the reading beam on the tag. In practical chemical and biological implementations these tags will be read in microfluidic devices, where perfect alignment may not always be possible. We therefore have to consider whether we can still recover the pitches on a tag if it is at some non-normal orientation with respect to the reading beam. A tag can rotate about three possible axes, as shown in fig. 6(A), from which we define three rotation angles $\eta, \theta$ and $\gamma$. Non-normal incidence requires a modification of the diffraction equations, so that a beam at diffracted angle $\alpha$ to the z-axis (see fig. 6(A) for axis definitions) satisfies $a(\cos \theta \sin \eta-\sin \alpha)=$ $m \lambda$, resulting in a distortion of the diffraction pattern.

The results of calculating the diffraction patterns modified by tag rotation are shown in fig. $6(\mathrm{~B}, \mathrm{C}$ and $\mathrm{D})$. A grating with a $1 \mu \mathrm{m}$ pitch is chosen to show the variation of $\alpha$ for example $m=+1$ and $m=-1$ orders, due to rotation $\eta$ (fig. $6(\mathrm{~B})$ ). Rotation by angle $\eta$ produces reduction of $\alpha$ on one side of the $m=0$ beam and an increase on the other: this increase in alpha could eventually 


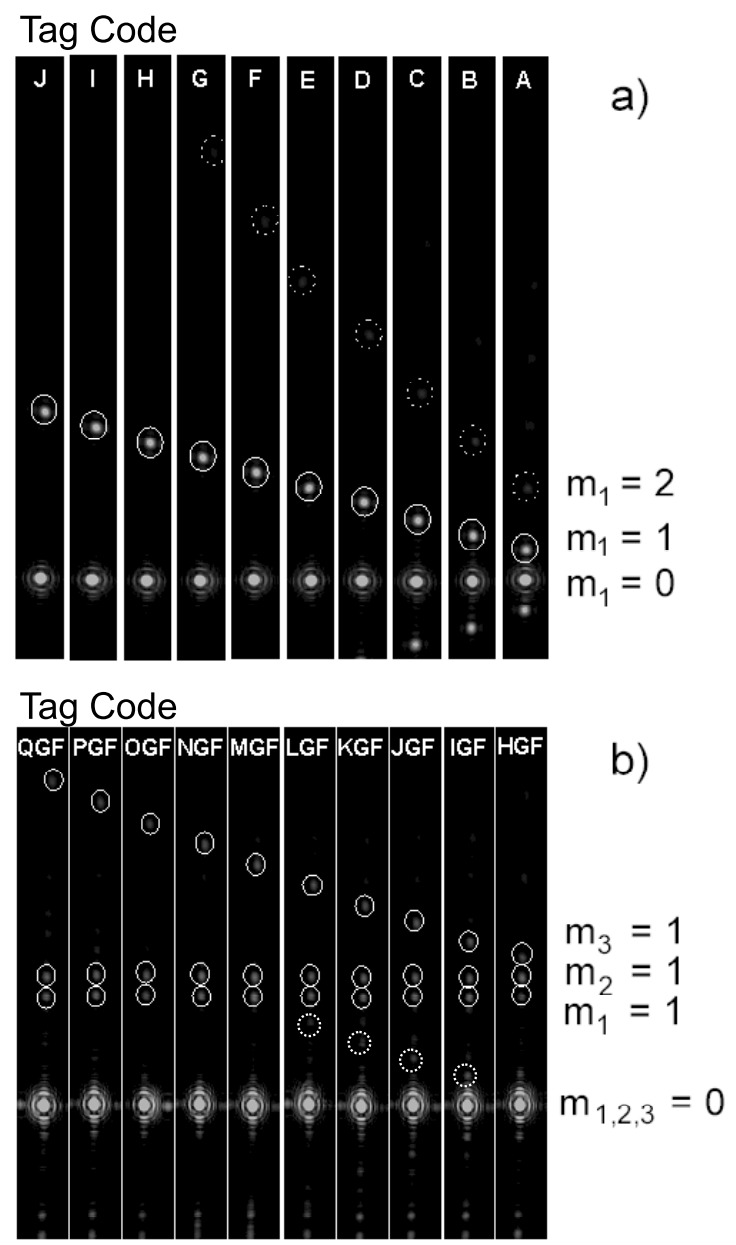

Fig. 5. Diffraction patterns created by a single grating tag (a), and tags containing three different gratings (b). Moving from left to right shows how a progressive decrease in the pitch of one of the gratings changes the diffraction pattern. Ghost beams are visible in dotted circles in (b).

cause the diffracted first order to move outside the $\pm 90^{\circ}$ detection range, if $\eta$ becomes very large. For the other two rotation axes, the diffracted spots change position, but $\alpha$ remains constant. The modified patterns produced by rotations $\gamma$ and $\theta$ have diffraction angles $\alpha$ which are no longer completely in the $x-z$ plane, so we define $\alpha_{x}$ and $\alpha_{y}$ to be the diffracted angles seen in the $x-z$ and $y-z$ planes, respectively, in order to show how the pattern appears in angular space. The large dots in fig. $6(\mathrm{C}$ and $\mathrm{D})$ show how the first order beams from an example $1 \mu \mathrm{m}$ pitch vary due to these rotations. The small dots are plots of beams with values of $\alpha$ between zero and $90^{\circ}$, in steps of $2^{\circ}$, and illustrate the shape of the modified diffraction patterns over a wide angular range. The open circle in fig. $6(\mathrm{C}$ and $\mathrm{D})$ shows the position of the $m=0$ beam. Rotation of the tag by angle $\gamma$ produces a rotation of the diffraction pattern about the zero order beam (fig. $6(\mathrm{C})$ ), with the rotation angle of the pattern equal to $\gamma$. Rotation by angle $\theta$ results in curvature of the diffraction pattern, which becomes greater with increasing $\theta$ (fig. 6(D)). 
It is, however, still perfectly possible to recover the pitch of a grating from a pattern distorted by tag rotation. To do this, we need to remove the unknown $\cos \theta \sin \eta$ from the diffraction equation. We can do this by measuring the absolute angle of both the $m=1$ and $m=-1$ diffracted beams with respect to the zero order, denoted $\alpha_{1}$ and $\alpha_{2}$, respectively. The pitch of the grating can then be reconstructed using $a=\frac{2 \lambda}{\sin \alpha_{2}-\sin \alpha_{1}}$, thus the tag can still be identified.
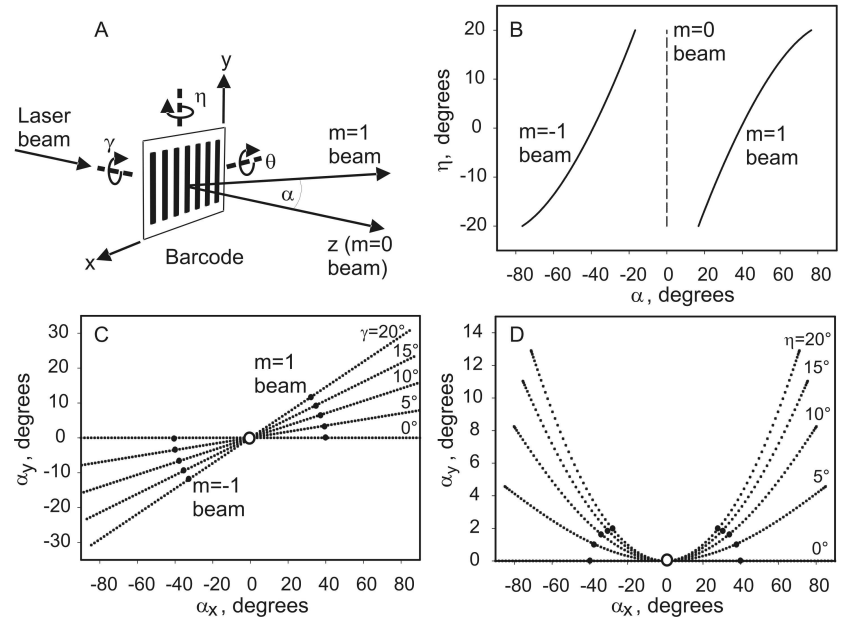

Fig. 6. Diffraction pattern distortions produced by tag rotation. A) The coordinate system used for distortion analysis. B) Variation of diffracted angle $\alpha$ for example $m=+1$ and $m=-1$ orders from grating with $a=1 \mu \mathrm{m}$, due to the rotation $\eta$. C) Variation in diffraction pattern orientation for selected values of $\gamma$ (given above corresponding curve). D) Variation of diffraction pattern shape for selected values of $\theta$ (given above corresponding curve).

The analysis given so far also assumes that only the first order diffraction spots encoding the data are present. In practice, however, this would only be true of a grating with a sinusoidal transmission function (fig. 7(A) inset), varying between zero transmission (perfectly opaque) and a maximum (perfectly transparent, denoted "1" fig.7). Such a grating has only a single frequency Fourier component in addition to the zero frequency, resulting in a diffraction pattern containing only zero and first order beams, as shown in fig. 7(A). The gratings we are considering, of the type shown in fig. 1, have a step transmission function, as seen in fig. 7(B and $\mathrm{C}$ ) insets. In addition to the fundamental Fourier component with period $a$, there are many higher frequency Fourier components which make up such a step function. These components appear as higher order beams $\left(m_{1}>1\right)$ in the grating's diffraction pattern, shown in fig. $7(\mathrm{~B}$ and $\mathrm{C})$. The relative intensity of the various higher diffracted orders, as calculated from Fraunhofer diffraction theory, depends upon the exact profile of the grating, in particular on the ratio $a /(a-b)$, where $b$ is the width of the transparent grating elements. Each successive higher order is less intense than the previous one. Some of the higher orders may be entirely absent from the diffraction patterns produced by certain grating profiles; for example a grating with $a_{1} /\left(a_{1}-b_{1}\right)=2$ has no second order $\left(m_{1}=2\right)$ diffracted beam 
(fig. $7(\mathrm{~B}))$, while a grating with $a_{1} /\left(a_{1}-b_{2}\right)=3$ has no third order $\left(m_{1}=3\right)$ beam (fig. $7(\mathrm{C})$ ).

The importance of the higher order beams is that they can be confused with the first order beams, leading to misreading of the tag. It is therefore critical to be able to distinguish the first order diffracted beams from the higher order beams. This can be achieved by use of intensity discrimination of the higher order diffracted beams. The discrimination level is set to the level of the most intense higher order beam. Defining the calculated first order intensity as $I_{1}$, this level $S$ can be defined as $S=I_{2} / I_{1}$, where $I_{2}$ is the intensity of the second order beam. This level $S$ was calculated for ideal square profile gratings with different values of $a /(a-b)$. Gratings with $a /(a-b)=2$ require the lowest level of threshold, since one needs only to discriminate the third order beams, as described above: setting $S=0.05$ eliminates all higher diffraction order beams and provides error-free identification of the tag (see inset of Fig. 2). For all other values, the second order beams have to be discriminated, resulting in the increasing values of $S$ shown in fig. 2 inset.

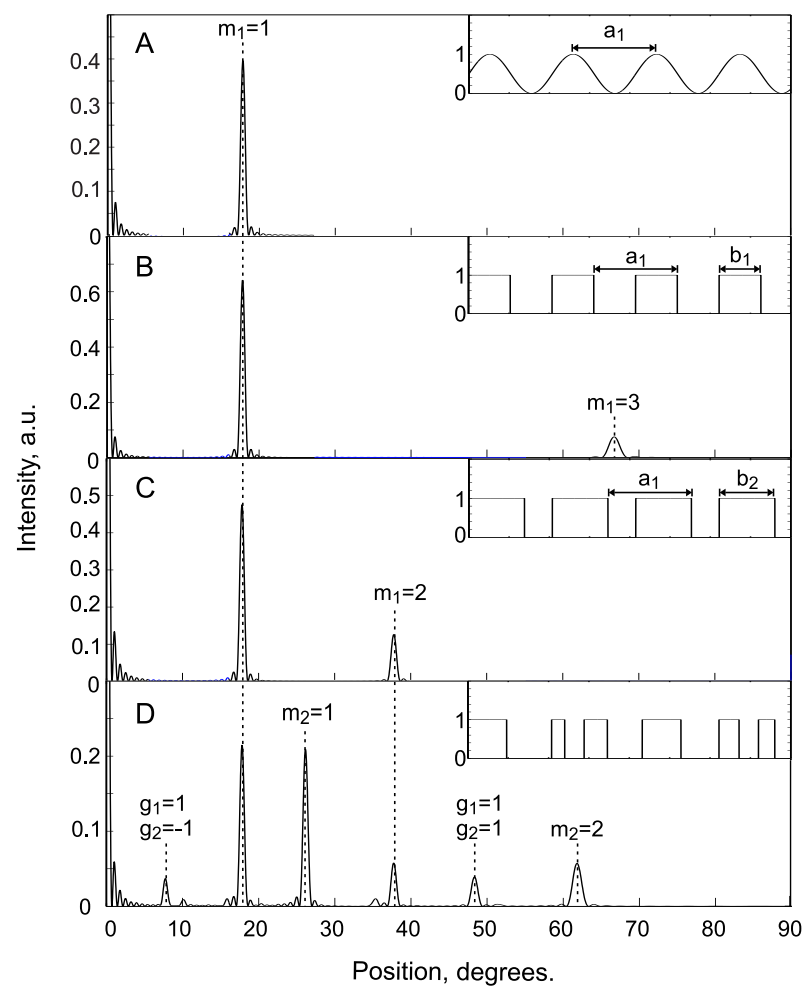

Fig. 7. Diffracted beams from various grating profiles, calculated by FFT. The grating transmission function is shown in the inset to each graph. A) Sinusoidal transmission function, only first order diffracted beam appears. B) Step transmission function with ratio $a_{1} /\left(a_{1}-b_{1}\right)=2$. C) Square profile with $a_{1} /\left(a_{1}-b_{2}\right)=3$. D) Grating with two superimposed pitches $a_{1}$ and $a_{2}$, both pitches have $a /(a-b)=3$. In addition to the higher order diffracted beams, ghost beams appear due to superimposing process. 
In addition to the higher order diffracted beams, when two or more pitches are superimposed, extra 'ghost' beams appear, which may also be confused with code first order beams. This is shown in fig. 1(D) for two superimposed pitches $a_{1}$ and $a_{2}$. These beams result from the fact that the superimposed grating profile (fig. 7(D) inset) is not an exact sum of the individual grating step functions. Therefore, in addition to the Fourier components of the individual step functions that make up the grating, the superimposed grating profile function has extra Fourier components that are linear combinations of the individual grating components (fig. 7(D)).

By performing Fast Fourier Transform (FFT) simulations of diffraction patterns from superimposed gratings, the positions of the ghost beams can be investigated. We find that each pair of pitches on a superimposed grating produces a set of ghost beams. The ghosts appear at angular positions that depend upon the spacing between the diffracted first order beams from each individual grating pitch. A pair of gratings superimposed as in fig. 1(D) will produce a diffraction pattern as in fig. 7(D), with ghost beams at positions $\alpha$ such that

$$
\sin \alpha=\lambda\left(\frac{g_{1}}{a_{1}}+\frac{g_{2}}{a_{2}}\right)
$$

where $g_{1}$ and $g_{2}$ can have the values $\pm 1, \pm 2, \ldots$ The metal-on-glass superimposed tags shown in fig. 4 display evidence of these ghost beams (see fig. 5(b), example ghosts shown in dotted circles). We can experimentally measure the ghost order positions and compare against equation (2), as shown in fig. 8. It can easily be seen that there is very good agreement between this equation and the measured diffracted spot positions.

In addition, we can obtain the intensities of the ghost beams from the FFT simulations, in order to determine whether they may be discriminated by a threshold level method, similar to that described for the higher order beams. The simulated intensities of ghost beams $g_{1}=g_{2}= \pm 1$, which are the most intense, are measured in regions well away from other features of significant intensity in the diffraction pattern. We find that if $I_{0}$ is the zero order intensity, $I_{1}$ is the first order intensity and $I_{g}$ the ghost order intensity, the relation $I_{1} / I_{0}=I_{g} / I_{1}$ appears to hold, with a tolerance of $\pm 2.4 \%$. We use the ratio $I_{g} / I_{1}$ as a discrimination level, in a similar manner to the value $S$ used for higher order elimination. From Fraunhofer diffraction theory, the ratio $I_{1} / I_{0}=$ $\left(\frac{a \sin \frac{b \pi}{a}}{b \pi}\right)^{2}$ decreases with increasing values of the ratio $a /(a-b)$ and is highest for $a /(a-b)=2$. The ratio $I_{g} / I_{1}$ behaves in the same manner, because of the equivalence $I_{1} / I_{0}=I_{g} / I_{1}$, as stated above. As we have already seen, the higher order intensity increases with increasing $a /(a-b)$. For $a /(a-b)>2$, the ghost intensities are less than the higher order beam intensities in the regions of the 
diffraction pattern considered here. Therefore in these regions, the higher order and ghost beams for gratings with $a /(a-b)>2$ are entirely discriminated by $S$. The optimal point for both ghost and higher order intensity is $a /(a-b)=3$ (either side of this value, one or the other increases) and we therefore chose this grating profile for subsequent investigation of the ghost orders. A similar analysis can be performed for other grating profiles.

There is an additional problem encountered when a ghost beam coincides in angular position with some other feature of significant intensity in the diffraction pattern. These features can include the side lobes of the zero order (seen near $0^{\circ}$ in fig. 7 ), which cause the ghost beam intensity to increase as its position approaches the zero order. Such ghost beams can be eliminated by having a discrimination threshold that increases close to the zero order.

A more drastic problem occurs when a ghost beam coincides with another ghost, or a higher order beam (shown in fig. 9). This creates a ghost with an intensity which is a large fraction of the first order intensity, which can therefore be extremely hard to discriminate from a code first order beam. It is difficult to remove such high intensity ghosts using a threshold method, so tags with high intensity ghosts have to be eliminated from the encoding library. By calculating all possible grating combinations for all different superposition levels, we can identify these tags and remove them from the possible set used for encoding, giving a set of revised values for the diffractive tag capacity (fig. 2 , dashed curves 2 - 5). We see, however, that the majority of the tags can still be used for encoding.

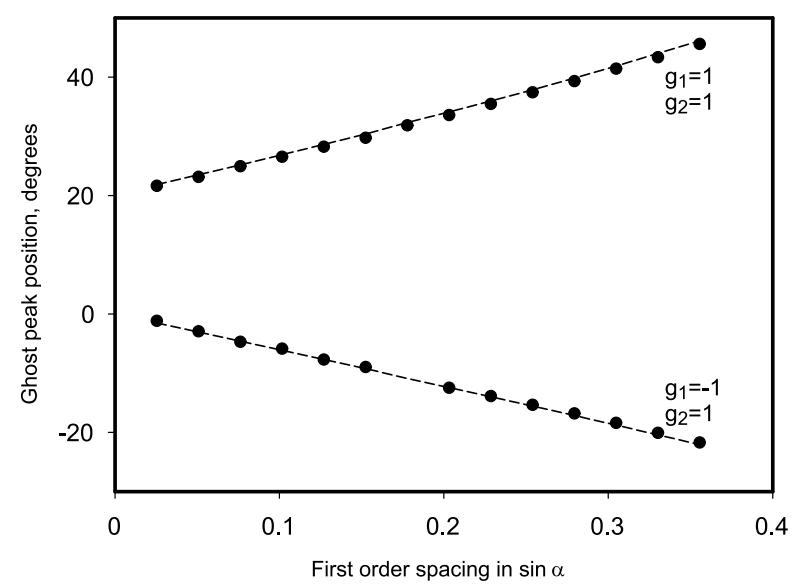

Fig. 8. Positions of the $g_{1}=1, g_{2}=1$ ghost peaks due to a pair of superimposed gratings. The position depends on the angular spacing between the first order beams from the gratings. Points are experimental measurements from the diffraction patterns of the metal-on glass gratings, dashed line is a plot of equation (2) for comparison. The equivalent curves for $g_{2}=-1$ are identical, but reflected about $0^{\circ}$ 


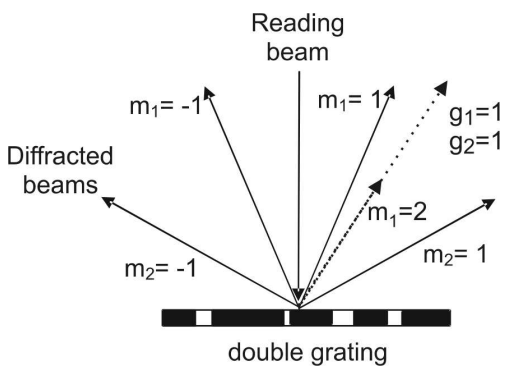

Fig. 9. An example of how a tag unreadable by a discrimination method is formed, when a 2nd order beam and a ghost beam coincide in angular position, producing a high intensity beam.

In conclusion we have demonstrated a new optical non-contact tagging technique based on superimposing large numbers of miniature diffraction gratings on a tag. We have also analyzed various limitations of the method for square profile gratings, such as higher order diffracted beams and ghost beams produced due to the superimposing process, which may be confused with code first order beams. However, we have shown that in most cases these beams can be eliminated by the use of an intensity threshold method. In addition, we have analyzed the robustness of the method with respect to non-normal incidence of the reading beam on the tag, which might be the case in applications. We have shown that it is still perfectly possible to read the tags with nonnormal incidence of the reading beam. With $50 \mathrm{~nm}$ nanofabrication resolution now routinely available the technique is capable of creating distinguishable tags containing at least 5 superimposed grating and encoding up to $10^{9}$ tags, each of which is only $100 \mu \mathrm{m}$ long and a few $\mu \mathrm{m}$ wide. To demonstrate this technique we manufactured a library of $50 \mu \mathrm{m} \times 50 \mu \mathrm{m}$ tags on a glass wafer. We have been able to demonstrate experimentally that the principle of superimposing works. With a nanofabrication minimum feature size of about $100 \mathrm{~nm}$ it has been possible to resolve tags containing at least three superimposed gratings providing capacity for more than 68,000 tags. An enormous increase in capacity is possible if two sets of mutually perpendicular gratings are used. Combinatorial analysis shows that up to $10^{21}$ different barcode tags can possibly be fabricated with such two-dimensional superimposed gratings up to order five. The robust nature of the tags, together with the non-contact remote reading capability makes them ideal for a large variety of biochemical, cytological, proteomic and genomic applications. These tags could also have widespread use in invisible marking for security applications and product marking, identification and tracking. 


\section{Acknowledgements}

The authors are grateful to Peter Roach, Cameron Neylon and Tony Bland for fruitful discussions, in particular on the tag requirements for biochemical applications. We thank Kelvin Nanotechnology, Glasgow for production of the barcode samples. We also acknowledge financial support from the EPSRC and the Basic Technology Research Programme of the UK Research Councils.

\section{References}

[1] Wilson, R., Cossins, A. R. and Spiller, D. G., Angew. Chem. Int. Ed. 45 (2006) 6104.

[2] Liu, L., Huang, Z. and Zhao, Y., Spectrochim. Acta A 62 (2005) 1039.

[3] Su, X., Zhang, J., Sun, L., Koo, T., Chan, S., Sundararajan, N., Yamakawa, M. and Berlin, A., Nano Lett. 5, (2005), 49.

[4] Pang, S., Smith, J., Onley, D., Reeve, J., Walker, M. and Foy, C., J. Immunol. Methods 302 (2005), 253.

[5] Evans, M., Sewter, C. and Hill, E., Assay Drug Dev. Technol. 1 (2003), 1,1 .

[6] Wang, S. X., Bae, S., Li, G., Sun, S., White, R. L., Kemp, J. T. and Webb, C. D., J. of Magn. and Magn. Mater. 293 (2005), 731.

[7] Jaiswal, J. K., and Simon, S. M., Trends Cell Biol. 14 (2004), 497.

[8] Mattheakis, L. C., Dias, J. M., Choi, Y., Gong, J., Bruchez, M. P., Liu, J. and Wang, E., E. Anal. Biochem. 327 (2004), 200.

[9] Seydack, M., Biosens. Bioelectron. 20 (2005), 2454.

[10] Reiss, B. D., Griffith Freeman, R., Walton, I. D., Norton, S. M., Smith, P. C., Stonas, W. G., Keating, C. D. and Natan, M. J., J. Electroanal. Chem. 522 (2002), 95.

[11] Breckmans, K., De Smedt, S. C., Roelent, C., Leblans, M., Pauwels, R. and Demeester, J., Nature Mat. 2 (2003), 169. 